\title{
The structure at $2 \AA$ resolution of Phycocyanin from Gracilaria chilensis and the energy transfer network in a PC-PC complex
}

\author{
Carlos Contreras-Martel ${ }^{\mathrm{b}}$, Adelio Matamala ${ }^{\mathrm{d}}$, Carola Bruna ${ }^{\mathrm{a}}$, German Poo-Caamaño ${ }^{\mathrm{c}}$, \\ Daniel Almonacid ${ }^{\mathrm{a}}$, Maximiliano Figueroa ${ }^{\mathrm{a}}$, José Martínez-Oyanedel ${ }^{\mathrm{a}}$, Marta Bunster ${ }^{\mathrm{a}, *}$ \\ a Laboratorio de Biofísica Molecular, Grupo de Biología Estructural, Depto. de Bioquímica y Biología Molecular, Facultad de Ciencias Biológicas, \\ Universidad de Concepción, Chile \\ b Institut de Biologie Structurale, CEA-CNRS-UJF, Grenoble, France \\ c Universidad del Bío-Bío, Concepción, Chile \\ d Laboratorio de Química Teórica y Computacional, Facultad de Ciencias Químicas, Depto. de Físico-Química, Universidad de Concepción, Chile
}

Received 5 August 2006; received in revised form 28 September 2006; accepted 29 September 2006

Available online 10 November 2006

\begin{abstract}
Phycocyanin is a phycobiliprotein involved in light harvesting and conduction of light to the reaction centers in cyanobacteria and red algae. The structure of C-phycocyanin from Gracilaria chilensis was solved by X-ray crystallography at 2.0 Å resolution in space group P $2{ }_{1}$. An interaction model between two PC heterohexamers was built, followed by molecular dynamic refinement. The best model showed an inter-hexamer rotation of $23^{\circ}$. The coordinates of a PC heterohexamer $(\alpha \beta)_{6}$ and of the PC-PC complex were used to perform energy transfer calculations between chromophores pairs using the fluorescence resonance energy transfer approach (FRET). Two main intra PC $\left({ }^{\mathrm{I}} \beta_{3}^{82} \rightarrow{ }^{\mathrm{I}} \alpha_{1}^{84} \rightarrow{ }^{\mathrm{I}} \alpha_{5}^{84} \rightarrow{ }^{\mathrm{I}} \beta_{6}^{82}\right.$ and $\left.{ }^{\mathrm{I}} \beta_{3}^{153} \rightarrow{ }^{\mathrm{I}} \beta_{5}^{153}\right)$ and two main inter $\mathrm{PC}\left({ }^{\mathrm{I}} \beta_{6}^{82} \rightarrow{ }^{\mathrm{II}} \beta_{3}^{82}\right.$ and $\left.{ }^{\mathrm{I}} \beta_{5}^{153} \rightarrow{ }^{\mathrm{II}} \beta_{3}^{153}\right)$ pathways were proposed based on the values of the energy transfer constants calculated for all the chromophore pairs in the hexamer and in the complex.
\end{abstract}

(C) 2006 Elsevier B.V. All rights reserved.

Keywords: Structure; Phycocyanin; Gracilaria chilensis; Protein-protein docking; Energy transfer pathway

\section{Introduction}

Phycobilisomes (PBS) are auxiliary photosynthetic complexes present in cyanobacteria and eukaryotic red algae. They are composed of phycobiliproteins (PBP) and linker polypeptides. Phycobiliproteins are highly fluorescent proteins due to the presence of covalently bonded chromophores to their cysteine residues, which provide them the functional properties to absorb light at a range of the visible spectrum not fully utilized by chlorophyll. Therefore, improving the light harvesting ability of the algae that contain them [1-3].

Phycobilisomes share a general common morphology [4] that consists of a core of face-to-back cylinders formed by stacked

\footnotetext{
is C.C.-M. and J.M.-O. contributed equally to this work.

* Corresponding author. Tel.: +56 41 203822; fax: +56 41239687.

E-mail address: mbunster@udec.cl (M. Bunster).
}

discs of PBP and several rods that radiate from the core composed of stacked back-to-back discs of PBP. Linker polypeptides are responsible for the maintenance of this structure, providing the necessary structural environment for efficient energy transfer and for the interaction with the photosynthetic reaction center $[1,5]$.

The phycobilisome of Gracilaria chilensis contains three phycobiliproteins: Phycoerythrin (PE), phycocyanin (PC) and allophycocyanin (APC) [6,7]. The absorption and emission spectra of these proteins overlap, which allows a non-radiative, direct and efficient transfer of the excitation energy among them $\left(\mathrm{PE} \lambda^{\mathrm{a}}{ }_{\max }=566 \mathrm{~nm} \quad \mathrm{PE} \lambda^{\mathrm{em}}{ }_{\max }=574 \mathrm{~nm} ; \mathrm{PC} \lambda^{\mathrm{a}}{ }_{\max }=621 \mathrm{~nm}\right.$ $\left.\mathrm{PC} \lambda \lambda_{\max }^{\mathrm{em}}=640 \mathrm{~nm} ; \mathrm{APC} \lambda_{\max }^{\mathrm{a}}=651 \mathrm{~nm} \mathrm{APC} \lambda_{\max }^{\mathrm{em}}=660 \mathrm{~nm}\right)$. This transfer is channeled along an energy gradient from the rods to the core and finally transferred to chlorophyll $a$ in the thylakoid membrane $[1,3]$.

In G. chilensis, the rods are composed of phycoerythrin and phycocyanin [6]. The three dimensional structure of phycoerythrin from this algae was reported in 2001 [7]. The structure of the 
next component of the rods, phycocyanin, is now presented in this paper. The structures of other phycocyanins from Fremyella diplosiphon (1cpc) [8], Spirulina platensis (1gho) [9], Synechococcus vulcanus (1i7y) [10,11] and Synechococcus elongatus (1jbo) [12] have been reported previously. The structure of phycocyanin from Polysiphonia ureceolata (1f99) [13] and Cyanidium caldarium (1phn) [14] have also been reported. These structures present a common subunit organization that consists of one $\alpha$ subunit (with cyanobilin (CB) associated to Cys84) and one $\beta$ subunit (with cyanobilins covalently attached to Cys82 and Cys153). They interact to form a heterodimer $(\alpha \beta)$ or a "pseudo monomer" that aggregates to form an hexameric ring $(\alpha \beta)_{6}$ able to pile up to form the main frame of a rod.

The rods in phycobilisomes normally include two or more phycocyanin hexamers and in this alga there are also one or more PE hexamers. Theoretical and experimental studies have been reported regarding the light transfer among chromophores inside one hexameric ring [8,14-17], including models that represent the energy transfer between hexamers $[8,14,16]$. In these studies, preferential energy transfer pathways have been proposed from data calculated using the Förster resonance approach [18], considering only the chromophore-chromophore distances and a Förster radius of $50 \AA$ as approximation [8] or the orientation among the transition dipole moments in one trimer of phycocyanin $[19,20]$.

This paper reports the three dimensional structure of phycocyanin from $G$. chilensis, the building of a docking model of two PC hexamers refined by molecular dynamics and the determination of the constants for the energy transfer in resonance between pairs of chromophores. For the latter purpose the orientation factors between pairs of chromophores were calculated from the dipole moments of their aromatic portions. The transfer constants were used to propose preferential pathways for the light conduction intra and inter hexamers of phycocyanin.

\section{Materials and methods}

\subsection{Protein purification and crystallization}

Phycocyanin from $G$. chilensis was purified according to Gantt [21]. Phycobiliproteins were extracted from algae collected at Coliumo Bay, Dichato, after maceration of $\mathrm{N}_{2(1)}$-frozen algae, in $20 \mathrm{mM}$ phosphate buffer $\mathrm{pH}$ 7.0. The extract was fractionated with ammonium sulphate (30\% and $60 \%$ saturation). The pellet was dissolved in distilled water and dialyzed against $5 \mathrm{mM}$ phosphate buffer $\mathrm{pH}$ 7.0. The phycobiliproteins were separated by anion exchange chromatography (Fractogel EMD DEAE 650S) in an FPLC system (Merck-HITACHI) using $5 \mathrm{mM}$ phosphate buffer as an equilibrium buffer and eluting the proteins with a linear gradient from 5 to $300 \mathrm{mM}$ sodium phosphate $\mathrm{pH}$ 7.0. The phycobiliprotein content of the eluted fractions was analyzed by absorption at $566 \mathrm{~nm}, 621 \mathrm{~nm}$ and $651 \mathrm{~nm}$. The phycocyanin-rich fraction was dialyzed against $900 \mathrm{mM}$ phosphate $\mathrm{pH} 7.0$ to precipitate phycocyanin and separate it from other phycobiliprotein contaminants. The phycocyanin precipitate was dissolved in $1 \mathrm{ml}$ of $50 \mathrm{mM}$ phosphate buffer $\mathrm{pH}$ 7.0. The protein concentration was estimated by the absorption at $280 \mathrm{~nm}$ and the purity was assessed by $12.5 \%$ SDS-PAGE and absorption and emission spectra. Crystals were grown by the vapor-diffusion method using sitting-drop insets at $291 \mathrm{~K} .1 \mathrm{ml}$ of $50 \mathrm{mM}$ HEPES buffer $\mathrm{pH}$ 7.0, 1.2 $\mathrm{M}$ ammonium sulphate was used in the reservoir. The $10 \mu \mathrm{l}$ droplet contained reservoir solution and protein at a concentration of $18 \mathrm{mg} \mathrm{ml}^{-1}$ in a $1: 1$ ratio.

\subsection{Data collection, structure solution and refinement}

The data was collected at the IMCA-CAT Synchrotron in Argonne with an ADSQ detector with an exposition time of $10 \mathrm{~s}$ per frame $(\lambda=1.00 \AA)$ and processed with XDS [22]. The initial phases were obtained by Molecular Replacement $[23,24]$ in the program package CNS [25] using the structure of C-phycocyanin from Spirullina platensis (1gho) [9] as a searching model. Sessions of manual rebuilding with TURBO FRODO [26] and refinement with CNS were performed. Lateral chains were assigned according to the residual electron density maps and to multiple sequence alignment of homologous proteins. After several cycles of refinement in the resolution range 60$2.0 \AA$ the $R_{\text {work }}$ and $R_{\text {free }}$ converged. The stereochemistry was verified with PROCHECK [27] and the refined model was deposited in the Protein Data Bank (code 2bv8).

\subsection{Phycocyanin-phycocyanin interaction model}

The coordinates of $2 \mathrm{bv} 8$ were used for the docking procedure of two phycocyanin molecules performed with the program ZDOCK $[28,29]$, with $6^{\circ}$ angular steps. The docking models were scored by the program considering desolvation, electrostatic and hydrophobic contributions [30-32]. The models generated were then analyzed to select those in agreement with the reported electron micrographs images [4]. The selected models were characterized by the number of hydrophobic residues in the interface using the protein-protein interaction server [33] and by the number of H-bonds in the interface using the program HBPLUS [34]. The highest scored rigid model was refined by molecular dynamics using the Force Field OPLS/AA [35] with the program GROMACS [36], with which a simulated annealing protocol for $200 \mathrm{ps}$ produced the convergence of the system. The coordinates of the chromophores in the final PC-PC interaction model were used to evaluate the energy transfer constants.

\subsection{Energy transfer calculations}

Förster theory has been confirmed to be a good approximation to study the light transfer processes occurring in phycobilisomes $[19,20]$. Förster defines [18] a transfer constant $k_{D A}$ as a measure of the frequency of events of energy transfer between a Donor (D) and an Acceptor (A). By introducing appropriate constants, the Förster equation can be expressed as a product of four terms.

$k_{D A}=C \cdot G \cdot S \cdot I$

where $C$ is a collection of constants that considers a refraction index $n=1.567 \quad[19,20,37,38], S$ include the spectroscopic properties of the interacting chromophores, $I$ is the overlap 
MK T PI T E AI A S ADS QGRF L SN GEL N S I NGRY QRAT A S L A AR SL TS N MK T P L T E A V A A A D S Q GRF L SS T E I QT A F GRF R QA S A S L A A A K A L T E K MK T P L T E A I A A A D S $Q$ Q GRF L S N T E L Q V V N GR Y N R R A T S S L E A A

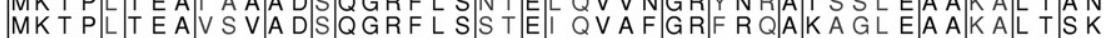

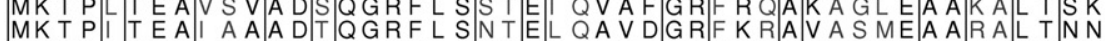

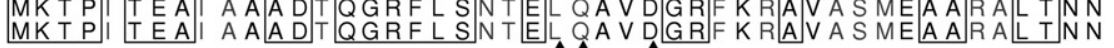

AERLIS GAAQSVYSKFPYT TQMQGP N Y A D T GKAKCARDI GYYLRM

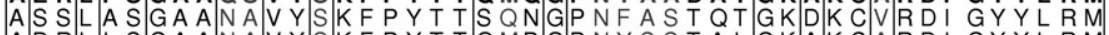
A D R L I S G A A N A V Y S K F P Y T T Q Q MP G P N Y S S T A I G K A K C A A R D I GY Y L R M

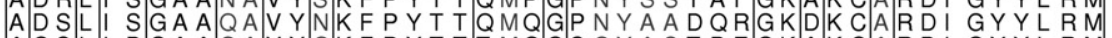

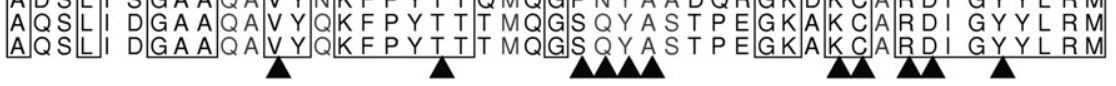

100

110

120

130

140

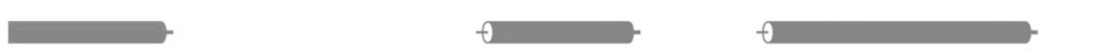

V T Y C L V V GA T GPMDE Y LI A GL DE I NR SFE L S P S WY I E A LE Y I K D S HD V T Y Y C L V G G T G P L D D Y L I G G I A E I N R T F D L S P S WY V E A L K Y I I K A N H G V T Y C L V V G G T GP M D D Y L V A GLL E E I N R T F E L S P S WY I E A L K Y I K N N H G V T Y C L I A G G T GP MDE Y L I A G I D E I N R T F E L S P S WY I E A L K Y I K I T Y C L V A G G T GP M D E Y L I A G L S E I N S T F D L S P S WY I E A L K Y I I K A N H G

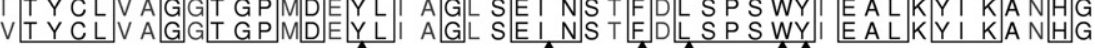

150

160

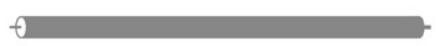

2BV8

LS GQAANE A NT YL D Y A N N L S

L S GDPA V E A NSYYI DYA I NAL S

L S G D V A N E A N T Y D D A I N T L S

L S G D A A

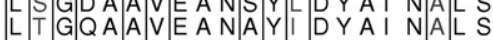

$1 \mathrm{GHO}$

$117 Y$

10

20

30

40

ML DAFA K V VA QADARGEF DS N T Q DALA L I AE GNKRLDI VNRTN N MLIDAFAKVVSQADARGEYLSGSQIDALSALVADGNKRMDVVNRIT GN MLL A F AKVVA QADARGEFLSNTQI DALLAIVSEGNKRLDVVNKI TN $M$ F D A F T K V V S Q A D T R GE ML S T A Q D D A L S Q MV A E SN K R L DVV N R I T S N

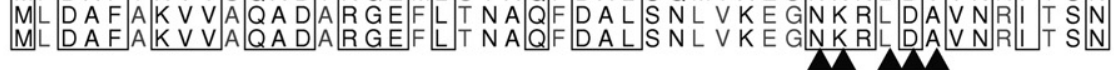

50

60

70

80

90

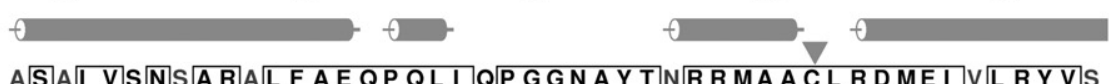

2BV8

ASA VSNS ARA LFAEQPQLIQPGGNAYTNRRMACLRDMEIV LRYVS SSTII VA N A A R SLLFEQPQLI A P GGNAYTSRRMAACLRDME I L RYV V

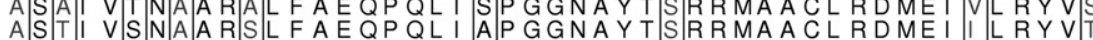
A ST VANAARALFAE QPQL Q PGGNAYTNRRMACLRDMEI LRYV

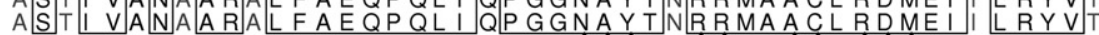

$117 Y$

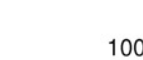

110

120

130

140

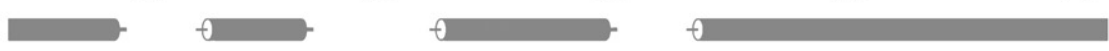

YAEI AGDSSVLD DRCLNGLRETYQALGTPGSSVAVAI E KMKEAS V D Y A I F A GDA S VL DDRCLNGL K E T Y L A L GTPGSS V A V G V QK MK D A A L A I Y A MI A GDA S VL DDR CLNGL RE T Y QA L GT P GAS V A V A I Q K MK D A A L A L Y A V F A GD A S V L E D R C L N GL RE T Y L A L GTP GSS S A V G G GIK MKE A A L A I

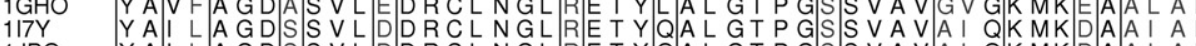

$1 \mathrm{JBO}$

150

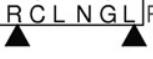

S $\mathrm{A}$.

QKMKDA A I A I

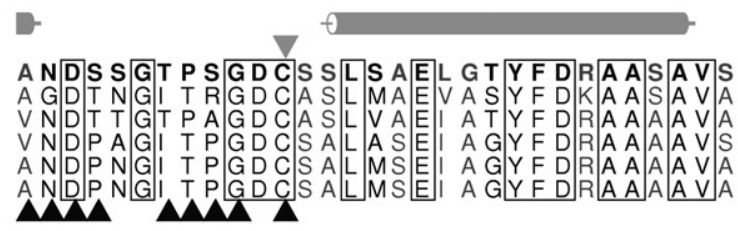


Table 1

Data collection, refinement and Ramachandran plot statistics

\begin{tabular}{|c|c|}
\hline \multicolumn{2}{|l|}{ Data collection } \\
\hline Space group & $\mathrm{P} 2_{1}$ \\
\hline \multicolumn{2}{|l|}{ Unit cell $(\AA ̊)$} \\
\hline $\mathrm{a}$ & 101.99 \\
\hline $\mathrm{b}$ & 151.80 \\
\hline $\mathrm{c}$ & 101.55 \\
\hline$\alpha=\gamma$ & 90.00 \\
\hline$\beta$ & 117.45 \\
\hline Resolution $(\AA ̊)$ & 2.0 \\
\hline Number of observed reflections & 178,325 \\
\hline Number of unique reflections & 172,985 \\
\hline Completeness (last bin) (\%) & $93.6(80.4)$ \\
\hline Rsym (last bin) (\%) & $6(30)$ \\
\hline $\mathrm{I} / \sigma(\mathrm{I})($ last bin) & $18.9(5.8)$ \\
\hline Last resolution shell & $2.0-2.12$ \\
\hline \multicolumn{2}{|l|}{ Refinement } \\
\hline Protein atoms & 15,024 \\
\hline Water molecules & 1,008 \\
\hline Heterogenic atoms & 780 \\
\hline$R_{\text {work }} / R_{\text {free }}$ & $19.9 / 23.1$ \\
\hline Average B-factor, protein $\left(\AA^{2}\right)$ & 28.4 \\
\hline Average B-factor, solvent $\left(\AA^{2}\right)$, & 53.4 \\
\hline 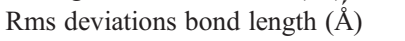 & 0.022 \\
\hline Rms deviations bond angle $\left(^{\circ}\right)$ & 2.3 \\
\hline Rms deviations dihedral angle $\left(^{\circ}\right)$ & 18.8 \\
\hline \multicolumn{2}{|l|}{ Ramachandran plot } \\
\hline Residues in core $(\%)$ & $1,707(95.2)$ \\
\hline Residues in additional allowed (\%) & $81(4.5)$ \\
\hline Residues in not allowed (\%) & $6(0.3)$ \\
\hline Non-glycine non-proline residues & 1,794 \\
\hline Total number of residues & 2,004 \\
\hline
\end{tabular}

integral between the emission and absorption spectra of the donor and acceptor chromophores respectively as described in [20]. Considering the homology among phycocyanins [39] and the structural similarity of the residues in contact with the chromophores (Fig. 1), the experimental values included in the terms $S$ and $I$ were assigned as those reported for Synechococcus sp PCC $7002[19,20]$. The geometric factor $G$,

$G=\frac{K_{D A}^{2}}{R_{D A}^{6}}$

includes the distances between the centers of mass for each pair of chromophores $R_{D A}$, and the dipole orientation coefficient $\kappa_{D A}$ as described in Eq. (3).

$\kappa_{D A}=\hat{\mu}_{D} \cdot \hat{\mu}_{A}-3\left(\hat{\mu}_{D} \cdot \hat{r}_{D A}\right)\left(\hat{\mu}_{A} \cdot \hat{r}_{D A}\right)$

where $\hat{\mu}_{D}$ and $\hat{\mu}_{A}$ are unit vectors describing the direction of the transition dipole moments of the donor and acceptor chromophores respectively and $\hat{r}_{D A}$ is the unit vector describing the

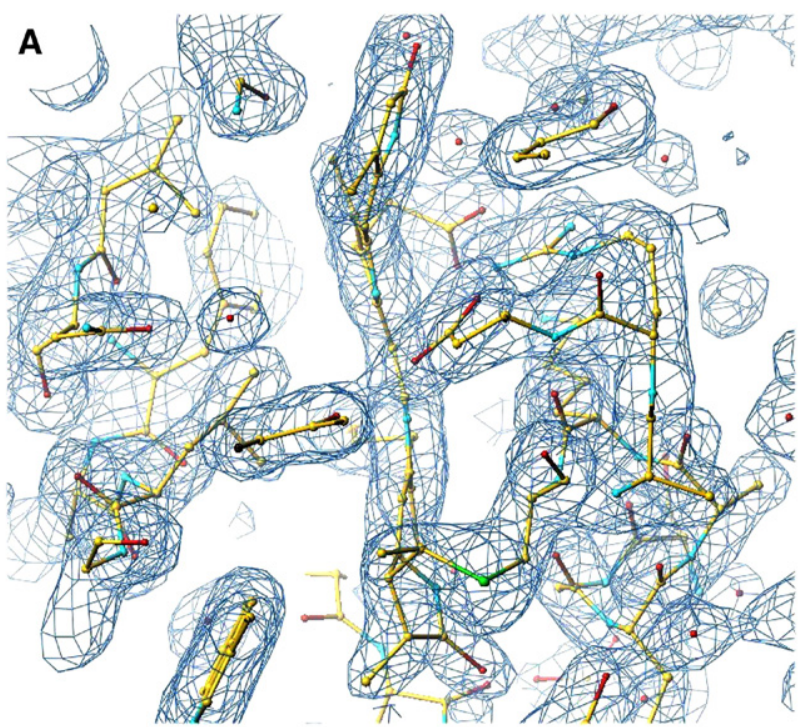

B

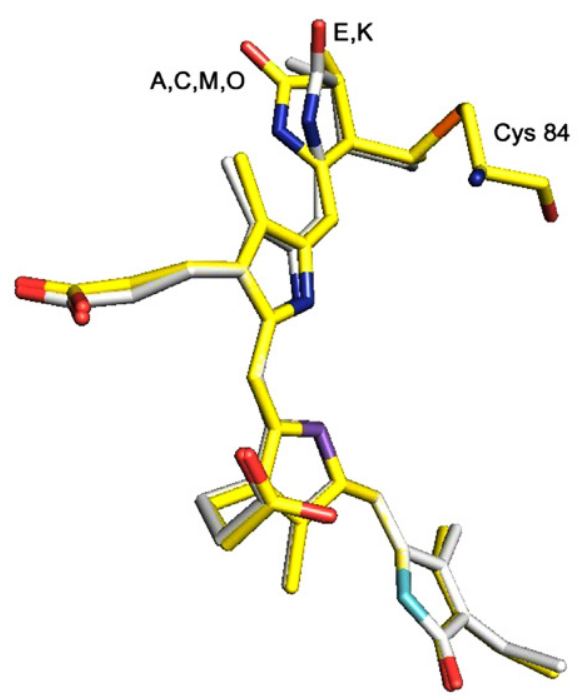

Fig. 2. Conformation of chromophores at $\alpha^{84}$. A) Fo-Fc electron density map showing the electron density of chromophore at $\alpha^{84}$ in chain K. B) Superposition of the chromophores at $\alpha^{84}$ in chains A (also representing chains $\mathrm{C}, \mathrm{M}$ and $\mathrm{O}$ ) and $\mathrm{E}$ (also representing chain $\mathrm{K}$ ).

direction of the line that connects the centers of mass of the interacting chromophores. The transition dipole moments were approximated to the dipole moments of the conjugated fragments [40] of the chromophores reported in 2 bv 8 and calculated by applying the semi empirical method PM3 Hamiltonian implemented in the software Gaussian98 [41]. Energy transfer steps with constants higher than $20 \mathrm{~ns}^{-1}$ and $10 \mathrm{~ns}^{-1}$ (transfer times shorter than $50 \mathrm{ps}$ and $100 \mathrm{ps}$ ) were used to define intra and inter phycocyanin preferential light transmission pathways respectively.

Fig. 1. Sequence and structural alignment of phycocyanin from G. chilensis. Sequence and structural alignment of $\alpha(\mathrm{A})$ and $\beta(\mathrm{B})$ subunits of C-phycocyanin from G. chilensis (2bv8), F. diplosiphon (1cpc), Polysiphonia urceolata (1f99), S. platensis (1gho), S. vulcanus (1i7y) and S. elongatus (1jbo). The rmsd in Å for the $\alpha$ and $\beta$ subunits on this comparison are respectively: $1 \mathrm{cpc}: 0.64-0.78$; $1 \mathrm{f} 99$ : $0.43-0.52 ; 1$ gho: $0.62-0.74 ; 1 \mathrm{i} 7 \mathrm{y}: 0.50-0.66$; $1 \mathrm{jbo}$ : $0.51-0.64$. The conserved regions are shown with squares. The conserved helical regions are indicated by cylinders drawn at the top of the alignment. The cysteines to which the chromophores are bound are indicated with a full grey inverted triangle. The conserved residues in contact with the chromophores in these structures are indicated by black triangles. 


\section{Results and discussion}

Although other phycobiliproteins are purified along with associated linker polypeptides [42], SDS-PAGE analysis of the purified phycocyanin of $G$. chilensis showed no presence of other polypeptides besides the $\alpha$ and $\beta$ subunits (data not shown).

Purple crystals appeared in one week and continued to grow for three weeks as rombohedral sheets of $0.1 \mathrm{~mm}$ high and $1 \mathrm{~mm}$ wide. The diffraction pattern of the crystals identified the $\mathrm{P} 22_{1}$ space group with $a=101.99 \AA, b=151.80 \AA, b=101.55 \AA$, $\alpha=\beta=90.00^{\circ}, \gamma=117.45^{\circ}$. The structure was solved as described in Materials and Methods, with data collection up to $2.0 \AA$ A. Results on the data statistics, refinement and stereochemistry are shown in Table 1 . The good quality of the molecular replacement allowed fitting the template (1gho) onto the electron density maps obtained and substituting the residues as required by the electron density. The lateral chains of highly conserved regions in the structures were maintained (Fig. 1). One hexamer $(\alpha \beta)_{6}$ was identified in the asymmetric unit. As non-crystallo-
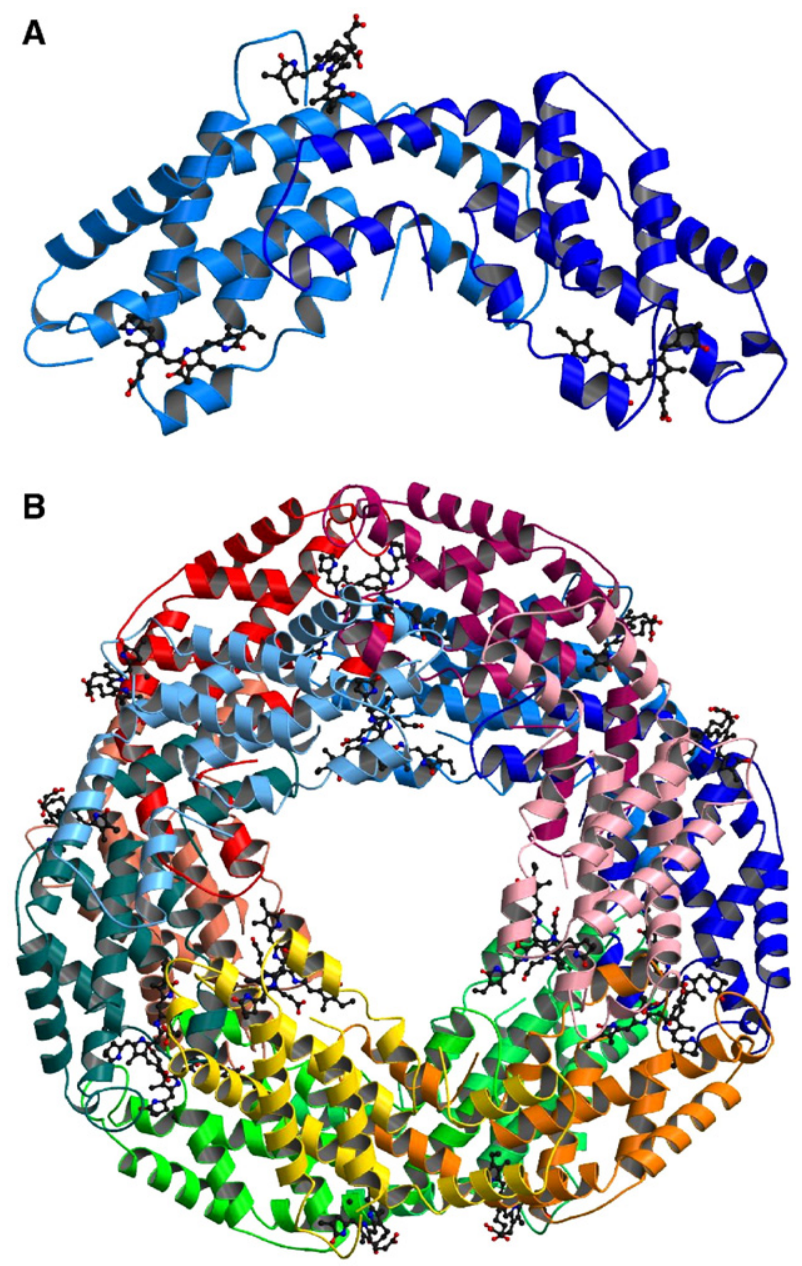

Fig. 3. Representation of the structure of phycocyanin from G. chilensis. A) Ribbon representation of the $\alpha$ (light blue) and $\beta$ (blue) subunits of C-phycocyanin of G. chilensis. The chromophores are shown in ball and stick representation. B) The molecule in the asymmetric unit. Ribbon representation of the heterohexamer $(\alpha \beta)_{6}$. The $\alpha$ subunits are chains A, C, E, K, M, O and the $\beta$ subunits are chains B, D, F, L, $\mathrm{N}, \mathrm{P}$ according to the structure deposited in the Protein Data Bank.

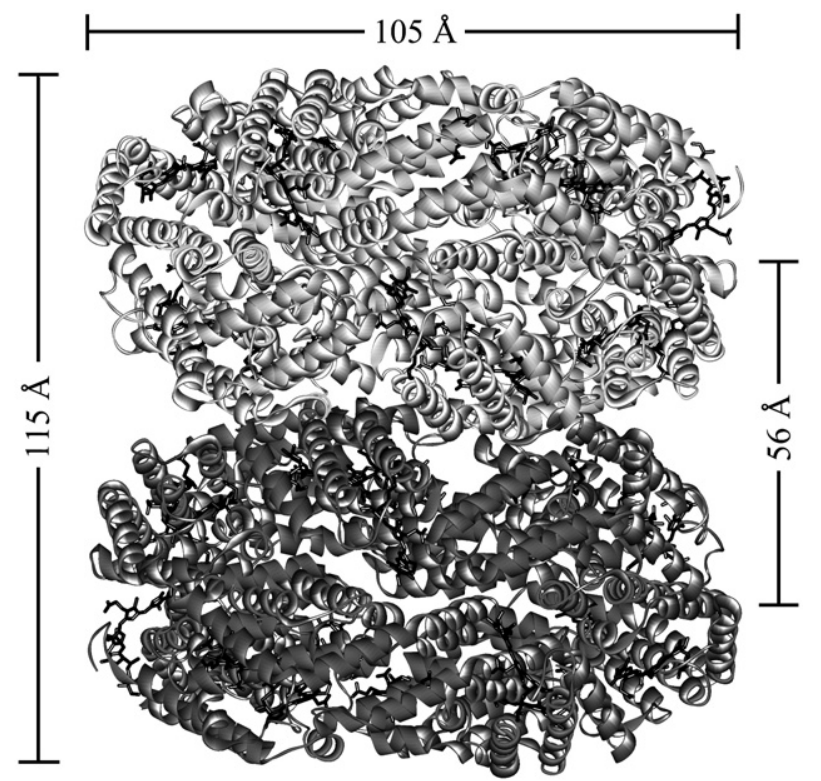

Fig. 4. PC-PC docking model. Ribbon representation of the refined PC-PC model. The general dimensions are indicated. The chromophores are shown in black.

graphic symmetry was not used, each subunit was solved independently 6 times providing confidence in the sequences reported for the $\alpha$ and $\beta$ subunits. The higher similarity obtained with BLAST [43] was, as expected, with PC from Gracilaria tenuistipitata. Nevertheless, there is also a clear sequence and structural similarity with all the other phycocyanins deposited at the Protein Data Bank, as it is shown on Fig. 1. The final model converged to a $R_{\text {work }}$ of $19.9 \%$ and $R_{\text {free }}$ of $23.1 \%$. The stereochemistry was also adequate and it is reported on Table 1, with the exception of $\mathrm{Thr}^{75}$, which occupied a disallowed region of the Ramachandran plot. This finding is present in all the structures of phycobiliproteins reported until now, regardless the type of phycobiliprotein or the space group. Asn 72 are methylated in all solved PC, including the one reported in this paper. The crystallographic data has been deposited at the Protein Data Bank under the accession code 2 bv8.

Phycocyanin from G. chilensis is formed by six $\alpha$ subunit (162 residues and one phycocyanobilin covalently bound to $\mathrm{C}^{84}$ ) and six $\beta$ subunit (172 residues and two phycocyanobilins associated to $\mathrm{C}^{82}$ and $\mathrm{C}^{153}$ ). Fig. $2 \mathrm{~A}$ shows the $2 \mathrm{Fo}-\mathrm{Fc}$ electron density map corresponding to phycocyanobilin associated to Cys 84 in chain $\mathrm{K}$. Both subunits contain a globulin like fold and they interact to form a heterodimer $(\alpha \beta)$ that oligomerises to a hexamer $(\alpha \beta)_{6}$ as is shown on Fig. 3. The structure allows the piling up and organization in phycobilisome rods. The hexamer is stabilized by hydrogen bonding and hydrophobic interactions. The structural analysis shows that $\mathrm{H}$-bonds that involve $\alpha$ subunits are participating in the stabilization of the hexamer and those between atoms of the $\beta$ subunits are responsible for the lateral stabilization of the trimer $(\alpha \beta)_{3}$. Aspartic acids $\left(\mathrm{D}_{\alpha}^{13}, \mathrm{D}_{\beta}^{13}, \mathrm{D}_{\beta}^{39}\right)$ interact with $\mathrm{A}$ and $\mathrm{D}$ rings of the respective chromophore. The involvement of Aspartic acids maintaining the curvature of phycobilins has been reported in all the phycobiliproteins $[7,44]$ and it may be a relevant feature for the biological activity. 
The docking procedure performed by ZDOCK [29] involved a rigid body interaction of two hexamers of phycocyanin (PC). The models generated were evaluated by the program using desolvation, electrostatic properties and hydrophobic interactions. The top models ranked by ZDOCK reproduced the piling of PBPs as proposed by electron micrographs of the rods [4]. Even though it has been reported that for surface recognition a rigid body approach is adequate, a molecular dynamics procedure with the whole structure was performed in order optimize contacts and to obtain better packed interaction surfaces [45]. The docking complex with the highest hydrophobic character in its interaction surface was refined by molecular dynamics as described in Materials and Methods. This produced modifications in the surfaces while preserving the relative position of the chromophores. The refined model presents a surface with less hydrophobic patches and an increased number of H-bonds ( 30 to 35 ) and salt bridges ( 3 to 7 ) between hexamers. The final complex is shown on Fig. 4. A rotation between hexamers was detected, which has previously been reported by Stec et al. [14] in a docking model of phycocyanin from $C$. caldarium. Thus, the best packing of the hexamers in a rod should be accomplished by a rotation of one over the other. This model allows a better packing of the side chains, increasing the number of interactions and improving the stability of the complex. This stable model was used to establish the coordinates of every chromophore for the determination of the energy transfer network in a PC-PC complex. Previous studies performed by our group [40] for phycocyanin from $F$. diplosiphon, show that a $20.5^{\circ}$ rotation not only improves the packing of hexamers in a $\mathrm{PC}-\mathrm{PC}$ complex, but also allows the participation of all the chromophores in the light transfer process, explaining the high efficiency of the system.

The calculation of $k_{D A}$ between all chromophores pairs was performed using the extended Eq. (1) with the orientation factors calculated from the dipole moments as described in Materials and Methods.

Our results confirm that the transfer constants are very dependent on the geometric and spectroscopic factor of the chromophores, but the dependence is stronger for the distances between chromophore pairs as it is shown on Table 2. At identical distances, the geometric factor becomes more important. Energy transfer steps for all chromophore pairs in our system were calculated and the value of the inverse of the transfer constant expressed in ps for the intra- or inter-phycocyanin steps are shown on Table 2. As hexamers of phycocyanin present high symmetry, in previous works, the analysis of possible light transfer pathways were performed in $1 / 3$ of the biological unit. In our case, as the structure was solved with one hexamer per asymmetric unit, slight differences in the conformation of equivalent chromophores were detected, specially for the chromophores at chain $\mathrm{E}\left(\alpha_{3}^{84}\right)$ and $\mathrm{K}\left(\alpha_{6}^{84}\right)$, which present a rotation of ring $\mathrm{D}$ as shown on Fig. 2B. This conformational diversity also contributes to the differences in the values of $k_{D A}$ or $k_{A D}$ for equivalent pairs of chromophores. The most significant differences are observed in the inter-hexamer steps of the pathway shown on Table 2. Differences of distances and orientation factors explain the different times obtained for equivalent steps.
Table 2

Characteristics of energy transfer steps between pairs of chromophores

Intrahexamer

\begin{tabular}{llllll}
\hline Pair & Distance $(\AA)$ & Angle $\left({ }^{\circ}\right)$ & Kappa & $1 / k_{D A}(\mathrm{ps})$ & $1 / k_{A D}(\mathrm{ps})$
\end{tabular}

Internal energy transfer pathways

$\begin{array}{llllll}\alpha_{1}^{84}-\beta_{3}^{82} & 20.6 & 61.33 & -1.26 & 6.27 & 4.07\end{array}$

$\begin{array}{llllll}\alpha_{3}^{84}-\beta_{2}^{82} & 20.5 & 93.81 & -1.17 & 7.17 & 4.66\end{array}$

$\begin{array}{llllll}\alpha_{2}^{84}-\beta_{1}^{82} & 20.7 & 64.70 & -1.27 & 6.39 & 4.15\end{array}$

$<\alpha^{84}-\beta^{82}>\quad 20.6$

$\alpha_{1}^{84}-\alpha_{5}^{84} \quad 25.6$

$\alpha_{3}^{84}-\alpha_{4}^{84} \quad 25.6$

$\alpha_{2}^{84}-\alpha_{6}^{84} \quad 25.6$

$<\alpha^{84}-\alpha^{84}>\quad 25.6$

$\alpha_{5}^{84}-\beta_{6}^{82} \quad 20.7$

$\alpha_{4}^{84}-\beta_{5}^{82} \quad 20.6$

$\alpha_{6}^{84}-\beta_{4}^{82} \quad 20.6$

$<\alpha^{84}-\beta^{82}>\quad 20.6$

66.75

6.61

4.29

16.90

$\begin{array}{llll}88.71 & 1.37 & 13.10 & 13.10\end{array}$

$\begin{array}{llll}81.03 & 1.26 & 15.00 & 15.00\end{array}$

$15.00 \quad 15.00$

$\begin{array}{llll}74.45 & -1.33 & 5.87 & 3.81\end{array}$

$\begin{array}{llll}64.13 & -1.34 & 5.48 & 3.56\end{array}$

$\begin{array}{llll}93.04 & -1.12 & 7.84 & 5.09\end{array}$

$6.39 \quad 4.15$

External energy transfer pathways

$\begin{array}{llllll}\beta_{1}^{153}-\beta_{6}^{153} & 26.2 & 158.08 & 1.52 & 5.63 & 5.63\end{array}$

$\begin{array}{llllll}\beta_{3}^{153}-\beta_{5}^{153} & 26.0 & 161.90 & 1.55 & 5.22 & 5.22\end{array}$

$\begin{array}{llllll}\beta_{2}^{153}-\beta_{4}^{153} & 26.1 & 162.65 & 1.50 & 6.06 & 6.06\end{array}$

$<\beta^{153}-\beta^{153}>\quad 26.1 \quad 5.63 \quad 5.63$

Interhexamer

\begin{tabular}{llllll}
\hline Pair & Distance $(\AA)$ & Angle $\left({ }^{\circ}\right)$ & Kappa & $1 / k_{D A}(\mathrm{ps})$ & $1 / k_{A D}(\mathrm{ps})$ \\
\hline
\end{tabular}

Internal energy transfer pathways

$\begin{array}{llllll}{ }^{\mathrm{I}} \beta_{4}^{82}-{ }^{\mathrm{II}} \beta_{1}^{82} & 22.02 & 72.24 & 0.98 & 12.67 & 12.67\end{array}$

$\begin{array}{llllll}{ }^{\mathrm{I}} \beta_{5}^{82}{ }^{\mathrm{II}} \mathrm{B}_{2}^{82} & 22.78 & 64.65 & 1.06 & 13.39 & 13.39\end{array}$

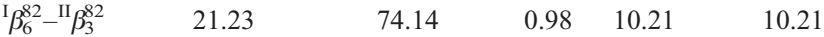

$<\beta^{82}{ }^{-1 \mathrm{I}} \beta^{82}>\quad 22.03 \quad 12.09 \quad 12.09$

External energy transfer pathways

$\begin{array}{llllll}{ }^{\mathrm{I}} \beta_{4}^{153}-{ }^{\mathrm{II}} \beta_{2}^{153} & 39.37 & 151.30 & 1.47 & 65.30 & 65.30\end{array}$

$\begin{array}{llllll}{ }^{\mathrm{I}} \beta_{5}^{153}-{ }^{\mathrm{II}} \beta_{3}^{153} & 38.38 & 148.87 & 1.49 & 57.15 & 57.15\end{array}$

$\begin{array}{llllll}{ }^{\mathrm{I}} \beta_{6}^{153}-{ }^{\mathrm{II}} \beta_{1}^{153} & 36.91 & 147.15 & 1.51 & 46.32 & 46.32\end{array}$

$<^{\mathrm{I}} \beta^{153}-{ }^{\mathrm{II}} \beta^{153}>38.22 \quad 56.26 \quad 56.26$

Other possible connections

\begin{tabular}{|c|c|c|c|c|c|}
\hline${ }^{\mathrm{I}} \alpha_{4}^{84}-{ }^{\mathrm{II}} \beta_{2}^{82}$ & 33.20 & 13.04 & -1.65 & 41.93 & 64.55 \\
\hline${ }^{\mathrm{I}} \alpha_{5}^{84}-{ }^{81} \beta_{3}^{82}$ & 31.66 & 10.21 & -1.75 & 28.02 & 43.19 \\
\hline${ }^{\mathrm{I}} \alpha_{6}^{84}-{ }^{\mathrm{II}} \beta_{1}^{82}$ & 32.16 & 29.92 & -1.86 & 27.18 & 41.86 \\
\hline$<^{\mathrm{I}} \alpha^{84}-{ }^{\mathrm{II}} \beta^{82}>$ & 32.34 & & & 33.71 & 49.85 \\
\hline${ }_{4}^{84}-{ }^{\mathrm{II}} \alpha_{3}^{84}$ & 31.57 & 76.12 & 1.34 & 47.52 & 47.52 \\
\hline${ }_{5}^{84}-{ }^{\mathrm{II}} \alpha_{1}^{84}$ & 29.83 & 96.98 & 1.45 & 29.19 & 29.19 \\
\hline${ }^{\mathrm{I}} \alpha_{6}^{84}-{ }^{\mathrm{II}} \alpha_{2}^{84}$ & 31.18 & 98.73 & 1.47 & 36.62 & 36.62 \\
\hline$<\alpha^{\mathrm{I}}{ }^{84}-{ }^{\mathrm{II}} \alpha^{84}>$ & 30.86 & & & 37.77 & 37.77 \\
\hline${ }^{\mathrm{I}} \beta_{4}^{82}-{ }^{\mathrm{II}} \alpha_{2}^{84}$ & 32.64 & 10.94 & -1.72 & 53.49 & 34.74 \\
\hline${ }^{\mathrm{I}} \beta_{5}^{82}-{ }^{\mathrm{II}} \alpha_{3}^{84}$ & 32.81 & 11.96 & -1.63 & 61.36 & 39.85 \\
\hline${ }^{\mathrm{I}} \beta_{6}^{82}-{ }^{\mathrm{II}} \alpha_{1}^{84}$ & 31.60 & 29.28 & -1.89 & 36.53 & 23.73 \\
\hline${ }^{-1} \beta^{82}-{ }^{I I} \alpha^{84}>$ & 32.35 & & & 50.46 & 32.77 \\
\hline
\end{tabular}

Distance: Distance between the donor acceptor mass centers, Angle: Measured between the dipolar moments, Kappa: Geometric factor, $1 / k_{D A}$ : Donor-Acceptor transfer rate, $1 / k_{A D}$ : Acceptor-Donor transfer rate. The identity of the hexamers of the PC-PC complex is indicated by the roman numbers in superscripts: I for the upper hexamer and II for the lower hexamer.

These effects may be additive considering that the PC-PC complex is the result of a docking procedure.

The analysis of the energy transfer constants suggests two main intra-phycocyanin pathways: ${ }^{\mathrm{I}} \beta_{3}^{82} \rightarrow{ }^{\mathrm{I}} \alpha_{1}^{84} \rightarrow{ }^{\mathrm{I}} \alpha_{5}^{84} \rightarrow{ }^{\mathrm{I}} \beta_{6}^{82}$ and ${ }^{\mathrm{I}} \beta_{3}^{153} \rightarrow{ }^{\mathrm{I}} \beta_{5}^{153}$ (these pathways are reproduced in each third of the hexameric ring in equivalent subunits). Two preferential 
A

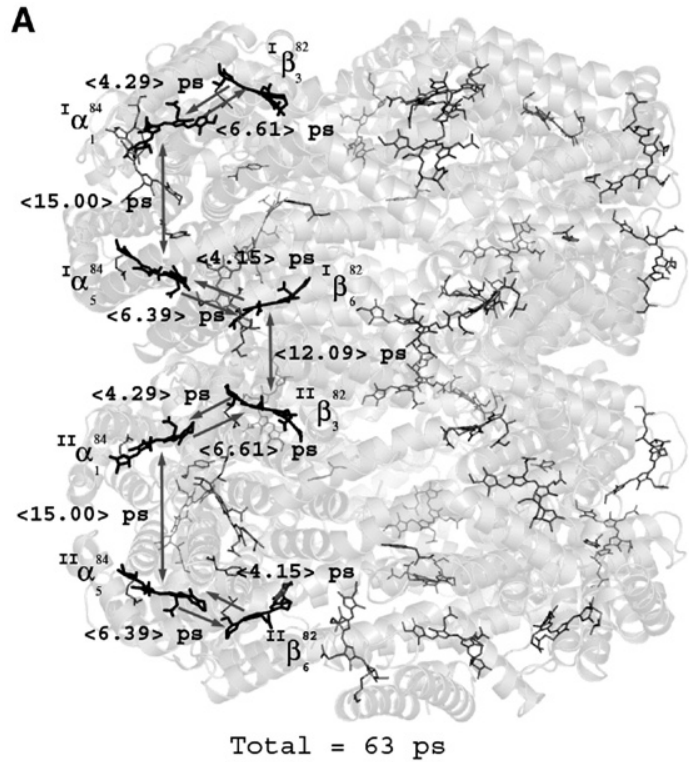

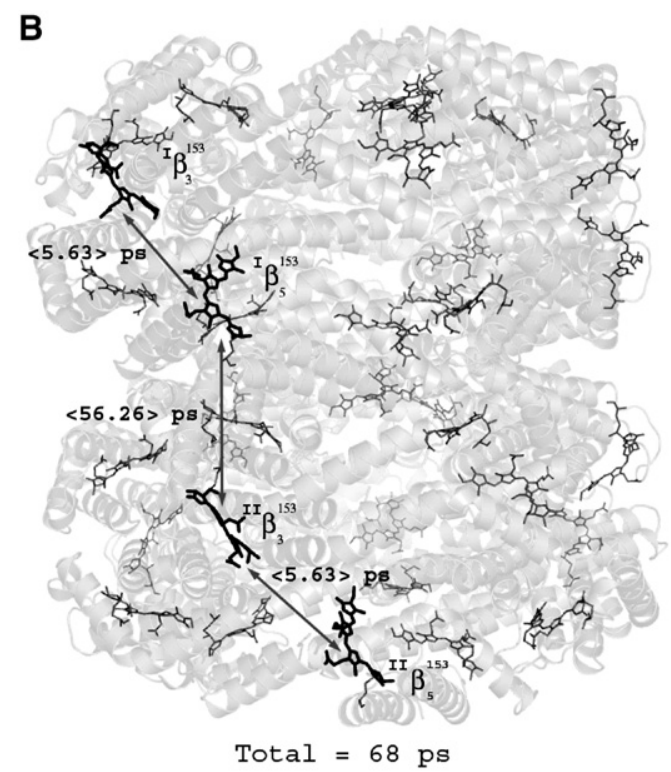

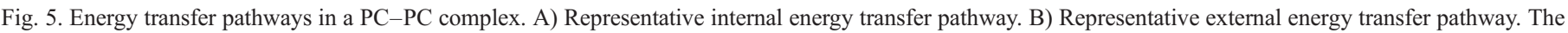

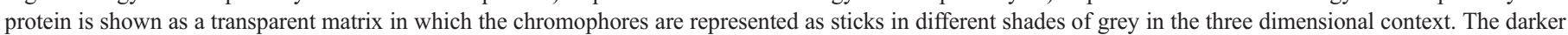
chromophores show the pathways. The average acceptor-donor transfer rates for each pair of chromophores are indicated.

energy transfer pathways from one PC hexamer to the next are also described ${ }^{\mathrm{I}} \beta_{6}^{82} \rightarrow{ }^{\mathrm{II}} \beta_{3}^{82}$ and ${ }^{\mathrm{I}} \beta_{5}^{153} \rightarrow{ }^{\mathrm{II}} \beta_{3}^{153}$. These pathways are indicated on Fig. 5. The total average transference time of the internal and external preferential pathways shown in Fig. 5 is 63 ps and 68 ps respectively. Experimental values in the range of 1 ps to $20 \mathrm{ps}$ for the excitation transfer between trimers and 45 to 130 ps between hexamers in a rod have been reported [15,20,46,47]. In addition, calculated values using comparable approaches in other C-PC show a similar ps timescale $[14,16]$.

Most of the calculations performed with phycocyanin hexamers indicate that the transfer between trimers in one phycocyanin ring occurs through $\alpha^{84}$ and identify $\beta^{82}$ as the donor between two PC hexamers. A complementary external pathway can be also described through $\beta^{153}$. As the chromophore groups are distributed along the antenna in the biological structure, every chromophore will receive light continuously either from other chromophores or from the environment, which validates the pathways through $\beta^{153}$.

\section{Conclusions}

Literature describes the analysis of the energy transfer pathways using heterodimers $(\alpha \beta)$ trimers $(\alpha \beta)_{3}$ or subcomplexes of phycobilisomes. In cases where the hexamer was used, it was built using crystallographic symmetry. In this article, the hexameric structure of phycocyanin from $G$. chilensis was solved experimentally. As the asymmetric unit was the hexamer, it was possible to detect slight differences between equivalent subunits, representing more accurately the biological functional unit.

The structure allowed to build a reliable model of the PC-PC complex, a minimum unit of a rod in phycobilisomes and to describe the light transfer pathways along the rod. The theoretical approach used the Förster equation, previously validated for monomers and trimers, was applied to the higher complex PC-PC, describing two main transfer pathways: An internal pathway of 63 ps that involves $\beta^{82}$ and $\alpha^{84}$ and an external of 68 ps that involves $\beta^{153}$.

The existence of two preferential independent pathways, which repeat three times within the complex, explains the high efficiency of the energy transfer in the phycobilisome. This assures that every chromophore that absorbs light has an acceptor, minimizing energy loss, constituting one of the designs of nature that could be imitated for biotechnological purposes.

\section{Acknowledgements}

We thank Jorge L. Ríos for his help with the data collection. This work was supported by Dirección de Investigación, Universidad de Concepción, DIUC Grant N²05.036.002-1.0.

\section{References}

[1] A.N. Glazer, Phycobilisomes, structure and dynamics, Annu. Rev. Microbiol. 36 (1982) 173-198.

[2] C. Lipschutz, E. Gantt, Association of Phycoerythrin and Phycocyanin: in vitro formation of a functional energy transferring phycobilisome complex of Porphyridium sordidum, Biochemistry 20 (1981) 3371-3376.

[3] D. Lundell, A.N. Glazer, Molecular architecture of a light harvesting antennae. Core substructure in Synechococcus 6301 phycobilisomes: two new allophycocyanins and allophycocyanin B complexes, J. Biol. Chem. 258 (1983) 902-908.

[4] A. Ducret, W. Sidler, E. Wehrli, G. Frank, R. Huber, Isolation, characterization and electron microscopy analysis of a hemidiscoidal phycobilisome type from the cyanobacteria Anabaena sp PCC7120, Eur. J. Biochem. 236 (1996) 1010-1024. 
[5] N. Tandeau de Marsac, G. Cohen-Bazire, Molecular composition of cyanobacterial phycobilisomes, Proc. Natl. Acad. Sci. U. S. A. 74 (4) (1977) 1635-1639.

[6] M. Bunster, J. Tellez, A. Candia, Characterization of phycobiliproteins present in Gracilaria chilensis, Bol. Soc. Chil. Quím. 42 (1997) 449-455.

[7] C. Contreras-Martel, J. Martínez-Oyanedel, M. Bunster, P. Legrand, C. Piras, X. Venerde, J.C. Fontecilla-Camps, Crystallization and $2.2 \AA$ resolution structure of R-phycoerythrin from Gracilaria chilensis: a case of a perfect hemihedral twinning, Acta Crystallogr., D Biol. Crystallogr. 57 (2001) $52-60$.

[8] M. Duerring, G.B Schmidt, R. Huber, Isolation, crystallization, crystal structure analysis and refinement of constitutive C-phycocyanin from the chromatically adapting cyanobacterium, Fremyella diplosiphon at $1.6 \AA$ resolution, J. Mol. Biol. 217 (1991) 577-592.

[9] X.-Q. Wang, L.-N. Li, W.R. Chang, D.C. Liang, Structure of C-phycocyanin from Spirulina platensis at $2.2 \AA$ resolution: a novel monoclinic crystal form for phycobiliproteins in phycobilisomes, Acta Crystallogr., D Biol. Crystallogr. 57 (2001) 784-792.

[10] N. Adir, Y. Dobrovetsky, N. Lerner, Structure of c-phycocyanin from the thermophilic cyanobacterium Synechococcus vulcanus at 2.5 A: structural implications for thermal stability in phycobilisome assembly, J. Mol. Biol. 313 (2001) 71-81.

[11] N. Adir, R. Vainer, V. Lerner, Refined Structure of C-phycocyanin from the Cyanobacterium Synechococcus vulcanus at $1.6 \AA$ : insights in the role of solvent molecules in thermal stability and co-factor structure, Biochim. Biophys. Acta 1556 (2002) 168-174.

[12] J. Nield, P.J. Rizkallah, J. Barber, N.E. Chayen, The $1.45 \AA$ three dimensional structure of C-phycocyanin from the thermophylic Cyanobacterium Synechococcus elongatus, J. Struct. Biol. 141 (2003) 149-155.

[13] W.R. Chang, T. Jiang, Z.L. Wang, Z.X. Yang, D.C. Liang, Crystal structure of R-phycoerythrin from Polisiphonia urceolata at $2.0 \AA$ resolution, J. Mol. Biol. 262 (1996) 721-731.

[14] B. Stec, R.F. Troxler, M.M. Teeter, Crystal structure of C-phycocyanin from Cyanidium caldarium provides a new perspective on phycobilisome assembly, Biophys. J. 76 (1999) 2912-2921.

[15] J. Zhang, F. Zhao, X. Zheng, H. Wang, Direct measurement of excitation transfer dynamics between two trimers in C-phycocyanin hexamer from Cyanobacterium Anabaena variabilis, Chem. Phys. Lett. 304 (1999) 357-364.

[16] A.A. Demidov, A.Y. Borisov, Computer simulation of energy migration on C-phycocyanin of the blue green algae Agmenellum quadruplicatum, Biophys. J. 64 (1993) 1375-1384.

[17] S.A. Pizarro, K. Sauer, Spectroscopic study of the Light harvesting protein C-phycocyanin associated with colorless linker peptides, Photochem. Photobiol. 73 (2001) 556-563.

[18] T. Förster, in: M. Florkin, E. Stotz (Eds.), Comprehensive Biochemistry, Mechanism of Energy Transfer, vol. 2, Elsevier, Amsterdam, 1967, pp. 261-280.

[19] M.-P. Debreczny, K. Sauer, J. Zhou, D.A. Bryant, Comparison of calculated and experimentally resolved rate constants for excitation energy transfer in C-phycocyanins, 1. Monomers, J. Phys. Chem. 99 (1995) 8412-8419.

[20] M.-P. Debreczny, K. Sauer, J. Zhou, D.A. Bryant, Comparison of calculated and experimentally resolved rate constants for excitation energy transfer in C-phycocyanins, 2. Trimers, J. Phys. Chem. 99 (1995) 8420-8431.

[21] E. Gantt, C.A. Lipschutz, Phycobilisomes from Porphyridium cruemtum, J. Cell Biol. 54 (1972) 313-324.

[22] W. Kabsch, Automatic processing of rotation diffraction data from crystals of initially unknown symmetry and cell constants, J. Appl. Crystallogr. 26 (1993) 795-800.

[23] M. Rossman, The molecular replacement method, Acta Crystallogr., A 46 (1990) 73-82.

[24] J. Navaza, AmoRe: an automated package for molecular replacement, Acta Crystallogr., A 50 (1994) 157-163.

[25] A.T. Brunger, P.D. Adams, G.M. Clore, W.L. Delano, P. Gros, R.W. GrosseKuntsleve, J.-S. Jiang, J. Kuszewski, N. Nilges, N.S. Pannu, R.J. Read, L.M. Rice, T. Simonson, G.L. Warren, Crystallography and NMR System (CNS): a new software system for macromolecular structure determination, Acta Crystallogr., D Biol. Crystallogr. 54 (1998) 905-921.
[26] A. Roussel, C. Cambillau, Silicon Graphics Geometry Partners Directory, The Turbo-Frodo Graphics Page, Silicon Graphics, Mountain View, CA, USA, 1991.

[27] R. Laskowsky, M. McArthur, D. Moss, J. Thornton, PROCHECK: a program to check the stereochemical quality of protein structures, J. Appl. Crystallogr. 26 (1993) 283-291.

[28] R. Chen, Z. Weng, Docking unbound proteins using shape complementarity, desolvation and electrostatics, Proteins 47 (2002) 281-294.

[29] R. Chen, L. Li, Z. Weng, ZDOCK: an initial stage protein docking algorithm, Proteins 52 (2003) 80-87.

[30] A. Archarov, V. Govorum, A. Dubanov, Y. Ivanov, A. Veselovsky, P. Lewi, O. Jansen, Protein-protein interactions as a target for drugs in proteomics, Proteomics 3 (2003) 380-391.

[31] B. Ma, T. Elkayam, H. Wolfson, R. Nussinov, Protein-protein interactions: structurally conserved residues distinguish between binding sites and exposed protein surfaces, Proc. Natl. Acad. Sci. U. S. A. 100 (2003) 5772-5777.

[32] B. Vallone, A. Miele, P. Vecchini, E. Chiancone, M. Brunori, Free energy of burying hydrophobic residues in the interface between protein subunits, Proc. Natl. Acad. Sci. U. S. A. 95 (1998) 6103-6107.

[33] S. Jones, J.M. Thornton, Progress in Biophysics and Molecular Biology, Protein-Protein Interactions: A Review of Protein Dimer Structures, vol. 63, 1995, pp. 31-165, http://www.biochem.ucl.ac.uk/bsm/PP/server/index. html.

[34] I.K. McDonald, J.M. Thornton, Satisfying hydrogen bonding potential in proteins, J. Mol. Biol. 238 (1994) 777-793.

[35] W.L. Jorgensen, J. Tirado-Rives, The OPLS force field for proteins. Energy minimizations for crystals of cyclic peptides and crambin, J. Am. Chem. Soc. 110 (1988) 1657-1666.

[36] W.F. Van Gunsteren, P.H. Hünenberger, A.E. Mark, P.E. Smith, I.G. Tironi, Computer simulation of protein motion, Comput. Phys. Commun. 91 (1995) 305-319.

[37] J. Grabowsky, E. Gantt, Photophysical properties of phycobiliproteins from phycobilisomes: fluorescent lifetimes quantum yields and polarization spectra, Photochem. Photobiol. 28 (1978) 39-45.

[38] F.J. Kleima, E. Hofmann, B. Gobets, I.H. van Stokkum, R. Grondelle, K. Diederichs, H. van Amerongen, Förster energy transfer in peridininchlorophyll-a-protein, Biophys. J. 78 (1) (2000) 344-353.

[39] K.E. Apt, J.E. Collier, A.R. Grossman, Evolution of phycobiliproteins, J. Mol. Biol. 248 (1995) 79-96.

[40] A.R. Matamala, D.E. Almonacid, M.F. Figueroa, J. Martínez-Oyanedel, M.C. Bunster, A Semiempirical Approach to the Intra-Phycocyanin and Inter-Phycocyanin FRET Pathways in Phycobilisomes, J. Comput. Chem. (2006) Manuscript accepted.

[41] M.J. Frisch, G.W. Trucks, H.B. Schlegel, G.E. Scuseria, M.A. Robb, J.R. Cheeseman, V.G. Zakrzewski, J.A. Montgomery Jr., R.E. Stratmann, J.C. Burant, S. Dapprich, J.M. Millam, A.D. Daniels, K.N. Kudin, M.C. Strain, O. Farkas, J. Tomasi, V. Barone, M. Cossi, R. Cammi, B. Mennucci, C. Pomelli, C. Adamo, S. Clifford, J. Ochterski, G.A. Petersson, P.Y. Ayala, Q. Cui, K. Morokuma, D.K. Malick, A.D. Rabuck, K. Raghavachari, J.B. Foresman, J. Cioslowski, J.V. Ortiz, A.G. Baboul, B.B. Stefanov, G. Liu, A. Liashenko, P. Piskorz, I. Komaromi, R. Gomperts, R.L. Martin, D.J. Fox, T. Keith, M.A. Al-Laham, C.Y. Peng, A. Nanayakkara, C. Gonzalez, M. Challacombe, P.M.W. Gill, B. Johnson, W. Chen, M.W. Wong, J.L. Andres, C. Gonzalez, M. Head-Gordon, E.S. Replogle, J.A. Pople, Gaussian 98, Revision A.7, Gaussian, Inc., Pittsburgh PA, 1998.

[42] W. Reuter, G. Wiegand, R. Huber, M. Than, Structural analysis at $2.2 \AA$ of orthorhombic crystals presents the asymmetry of allophycocyanin-linker complex AP-L $\mathrm{L}_{\mathrm{C}}^{7.8}$, from phycobilisomes from Mastigocladus laminosus, Proc. Natl. Acad. Sci. U. S. A. 96 (1999) 1363-1368.

[43] S.F. Altschul, W. Gish, W. Miller, E.W. Myers, D.J. Lipman, Basic local alignment search tool, J. Mol. Biol. 215 (1990) 403-410.

[44] J. Martínez-Oyanedel, C. Contreras-Martel, C. Bruna, M. Bunster, Structural-functional analysis of the oligomeric protein R-phycoerythrin, Biol. Res. 37 (2004) 733-745. 
[45] C.J. Camacho, Modeling side-chains using molecular dynamics improve recognition of binding region in CAPRI targets, Proteins 60 (2005) $245-251$.

[46] A.R. Holzwarth, J. Wendler, G.W. Suter, Studies on chromophore coupling in isolated phycobiliproteins: II. Picosecond energy transfer kinetics and time resolved fluorescence spectra of C-phycocyanin from Synechococcus 6301 as function of the aggregation state, Biophys. J. 51 (1987) 1-12.

[47] A.R. Holzwarth, Structure-function relationships and energy transfer in phycobiliprotein antennae, Physiol. Plant. 83 (1991) 518-528. 